ANNALES

POLONICI MATHEMATICI

$83.3(2004)$

\title{
Positive solutions with given slope of a nonlocal second order boundary value problem with sign changing nonlinearities
}

\author{
by P. Ch. Tsamatos (Ioannina)
}

\begin{abstract}
We study a nonlocal boundary value problem for the equation $x^{\prime \prime}(t)+$ $f\left(t, x(t), x^{\prime}(t)\right)=0, t \in[0,1]$. By applying fixed point theorems on appropriate cones, we prove that this boundary value problem admits positive solutions with slope in a given annulus. It is remarkable that we do not assume $f \geq 0$. Here the sign of the function $f$ may change.
\end{abstract}

1. Introduction. This paper discusses the nonlinear equation

$$
x^{\prime \prime}(t)+f\left(t, x(t), x^{\prime}(t)\right)=0, \quad t \in I:=[0,1],
$$

with the initial condition

$$
x(0)=0
$$

and the nonlocal boundary condition

$$
x^{\prime}(1)=\int_{0}^{1} x^{\prime}(s) d g(s),
$$

where $f: I \times \mathbb{R} \times \mathbb{R} \rightarrow \mathbb{R}$ is a continuous function, $g: I \rightarrow \mathbb{R}$ is an increasing function and the integral in (1.3) is a Riemann-Stieltjes integral.

Condition (1.3) is obviously the continuous version of the multipoint boundary condition

$$
x^{\prime}(1)=\sum_{i=1}^{m} \alpha_{i} x^{\prime}\left(\xi_{i}\right),
$$

where $\xi_{i} \in(0,1), i=1, \ldots, m$ and the real numbers $\alpha_{1}, \ldots, \alpha_{m}$ have the same sign. The boundary value problem (1.1), (1.2), (1.4) appeared early

2000 Mathematics Subject Classification: Primary 34B18; Secondary 34B10.

Key words and phrases: boundary value problem, positive sublinear solution, nonlocal boundary value problem. 
in the literature (see [16] and the references therein) and has recently been studied e.g. in $[13,14]$. Also the problem of the existence of positive solutions for the nonlocal boundary value problem (1.1)-(1.3) has been dealt with by Karakostas and Tsamatos in several papers [17-20]. We note that the interest in the existence of positive solutions for ordinary differential equations comes from the corresponding problems in partial differential equations. Indeed, the problem of the existence of positive solutions for partial semilinear elliptic equations is very old, has many applications and has been widely studied. (See the review article by Lions [23].) H. Wang [27] showed how such a problem can be reduced to a second order boundary value problem for ordinary differential equations with two-point boundary conditions. Since then, the problem of existence of positive solutions for second order ordinary differential equations, with various boundary conditions, has been dealt with by many authors. (See e.g. [1, 4-10, 12, 15, 17-20, 22, 24-26].) The book of Agarwal, O'Regan and Wong [2] gives a good overview on this issue.

A very common assumption in the study of positive solutions for boundary value problems for equation (1.1), especially when Krasnosel'skiı's fixed point theorem is applied, is that $f \geq 0$. (See e.g. [8-10, 15, 17-20, 22, 24-27].) This assumption is very helpful in proving that such a boundary value problem has at least one positive and concave solution. There are fewer papers in which $f$ is allowed to change sign. (See [1, 4, 5, 12, 22].) Motivated mainly by $[12,17-20,22]$, in the present paper we establish sufficient conditions under which the boundary value problem (1.1)-(1.3) has positive solutions in the case when $f$ can change sign. To obtain our results, apart from the well known Krasnosel'skil fixed point theorem (see Theorem 1.2 below), we also use another fixed point theorem on a Banach space ordered by an appropriate cone (see Theorem 1.1 below). The concept of a cone in a Banach space is essential to formulate and apply both these theorems.

Definition. Let $\mathcal{B}$ be a real Banach space. A cone in $\mathcal{B}$ is a nonempty, closed set $\mathbb{K} \subset \mathcal{B}$ such that

(i) $\kappa u+\lambda v \in \mathbb{K}$ for all $u, v \in \mathbb{K}$ and all $\kappa, \lambda \geq 0$,

(ii) $u,-u \in \mathbb{K}$ implies $u=0$.

In a Banach space $\mathcal{B}$ we set

$$
B_{\varrho}:=\{x \in \mathcal{B}:\|x\|<\varrho\}, \quad \partial B_{\varrho}:=\{x \in \mathcal{B}:\|x\|=\varrho\} .
$$

Theorem 1.1 ([3, p. 661]). Let $\mathcal{B}$ a Banach space, $\mathbb{K}$ a cone in $\mathcal{B}$ and $h: \mathbb{K} \cap \bar{B}_{\varrho} \rightarrow \mathbb{K}$ a compact map such that $h(x) \neq \lambda x$ for all $x \in \mathbb{K} \cap \partial B_{\varrho}$ and $\lambda \geq 1$. Then $h$ has a fixed point in $\mathbb{K} \cap \bar{B}_{\varrho}$.

Theorem $1.2([11,21])$. Let $\mathcal{B}$ be a Banach space and let $\mathbb{K}$ be a cone in $\mathcal{B}$. Assume that $\Omega_{1}$ and $\Omega_{2}$ are open bounded subsets of $\mathcal{B}$, with $0 \in \Omega_{1} \subset$ 
$\bar{\Omega}_{1} \subset \Omega_{2}$, and let

$$
T: \mathbb{K} \cap\left(\bar{\Omega}_{2} \backslash \Omega_{1}\right) \rightarrow \mathbb{K}
$$

be a completely continuous operator such that either

$$
\|T u\| \leq\|u\|, \quad u \in \mathbb{K} \cap \partial \Omega_{1}, \quad\|T u\| \geq\|u\|, \quad u \in \mathbb{K} \cap \partial \Omega_{2},
$$

or

$$
\|T u\| \geq\|u\|, \quad u \in \mathbb{K} \cap \partial \Omega_{1}, \quad\|T u\| \leq\|u\|, \quad u \in \mathbb{K} \cap \partial \Omega_{2} .
$$

Then $T$ has a fixed point in $\mathbb{K} \cap\left(\bar{\Omega}_{2} \backslash \Omega_{1}\right)$.

2. The assumptions and main results. We denote by $\mathbb{R}$ the real line and by $\mathbb{R}^{+}, I$ the intervals $[0, \infty),[0,1]$ respectively. Also, $C_{0}^{1}(I)$ denotes the space of all functions $x: I \rightarrow \mathbb{R}$ with $x^{\prime}$ continuous on $I$ and $x(0)=0$. The norm

$$
\|x\|_{0}^{1}:=\sup \left\{\left|x^{\prime}(t)\right|: t \in I\right\}
$$

makes $C_{0}^{1}(I)$ a Banach space.

For the function $g$ we assume the following:

$\left(H_{1}\right) \quad g: I \rightarrow \mathbb{R}$ is an increasing function such that

$$
g(0)=0, \quad g(1) \neq 1 .
$$

Consider equation (1.1) with the boundary conditions (1.2), (1.3). By a solution of the boundary value problem (1.1)-(1.3) we mean a function $x \in C_{0}^{1}(I)$ satisfying condition (1.3) and equation (1.1) for all $t \in I$.

Searching for solutions we shall reformulate the problem (1.1)-(1.3) to obtain an operator equation of the form $x=A x$, for an appropriate operator $A$. To find $A$ consider an equation of the form

$$
x^{\prime \prime}(t)+z(t)=0, \quad t \in I,
$$

subject to conditions (1.2), (1.3). By integration we get

$$
x^{\prime}(t)=x^{\prime}(1)+\int_{t}^{1} z(s) d s .
$$

Then, from (1.3), it follows that

$$
x^{\prime}(1)=\gamma \int_{0}^{1} \int_{t}^{1} z(s) d s d g(t),
$$

where

$$
\gamma:=\frac{1}{1-g(1)}
$$


Integrating (2.1) once again we obtain

$$
x(t)=\gamma t \int_{0}^{1} \int_{s}^{1} z(r) d r d g(s)+\int_{0}^{t} \int_{s}^{1} z(r) d r d s, \quad t \in I .
$$

This process shows that solving the boundary value problem (1.1)-(1.3) is equivalent to solving the operator equation $x=A x$ in $C_{0}^{1}(I)$, where $A$ is the operator defined by

$$
(A x)(t)=\gamma t \int_{0}^{1} \int_{s}^{1} f\left(r, x(r), x^{\prime}(r)\right) d r d g(s)+\int_{0}^{t} \int_{s}^{1} f\left(r, x(r), x^{\prime}(r)\right) d r d s
$$

for $x \in C_{0}^{1}(I)$ and $t \in I$.

Before presenting our results we state our assumptions as well as introduce some useful notations:

$\left(H_{2}\right) \quad$ There exist nonnegative real-valued functions $p, q, r$ in $L^{1}(I)$ and nonnegative, nondecreasing real-valued functions $\Phi, \Psi$, locally integrable on $\mathbb{R}^{+}$and such that

$$
|f(t, u, v)| \leq p(t)+q(t) \Phi(|u|)+r(t) \Psi(|v|)
$$

for all $(t, u, v) \in I \times \mathbb{R} \times \mathbb{R}$. Moreover, $p, q$ and $r$ are not equal to zero almost everywhere.

Next define the continuous functions

$$
\begin{gathered}
P(t):=\int_{t}^{1} p(s) d s, \quad Q(t):=\int_{t}^{1} q(s) d s, \quad R(t):=\int_{t}^{1} r(s) d s, \quad t \in I, \\
Q_{m}(t):=\int_{t}^{1} q(\theta) \Phi(\theta m) d \theta, \quad t \in I, m \in \mathbb{R}^{+},
\end{gathered}
$$

and

$$
F(m):=\gamma \int_{0}^{1} \int_{s}^{1} f(r, m r, m) d r d g(s), \quad m \in \mathbb{R}^{+} .
$$

Moreover, if $w: I \rightarrow \mathbb{R}$ is a continuous function, we define

$$
w_{g}=\int_{0}^{1} w(s) d g(s) \text {. }
$$

$\left(H_{3}\right) \quad$ There exists $T>0$ such that

$$
|\gamma|\left(P_{g}+\left(Q_{T}\right)_{g}+\Psi(T) R_{g}\right)+P(0)+Q_{T}(0)+R(0) \Psi(T)<T .
$$

$\left(H_{4}\right) \quad$ The function $\gamma f(t, u, v)$ is nonincreasing with respect to the variables $u, v$.

Now we are in a position to prove our first main result: 
THEOREM 2.1. Let assumptions $\left(H_{1}\right)-\left(H_{4}\right)$ be satisfied, and suppose that $\left(H_{5}\right) \quad$ there exists $\Theta>T$ such that $F(\Theta)-P(0)-Q_{\Theta}(0)-\Psi(\Theta) R(0) \geq 0$. Then there exists a solution $x \in C_{0}^{1}(I)$ of the boundary value problem (1.1)(1.3) such that $0 \leq x(t) \leq T t$ for $t \in I$, where $T$ is defined by $\left(H_{3}\right)$.

Proof. We intend to apply Theorem 1.1. For this purpose consider the set

$$
\mathbb{K}:=\left\{x \in C_{0}^{1}(I): x^{\prime}(t) \geq 0, t \in I\right\},
$$

which is a cone in $C_{0}^{1}(I)$, and the set

$$
B_{\Theta}=\left\{x \in C_{0}^{1}(I):\|x\|_{0}^{1}<\Theta\right\},
$$

where $\Theta$ is the positive constant ensured by assumption $\left(H_{5}\right)$. We will prove that

$$
A\left(\mathbb{K} \cap \bar{B}_{\Theta}\right) \subset \mathbb{K}
$$

Indeed, if $x \in \mathbb{K} \cap \bar{B}_{\Theta}$, then $0 \leq x^{\prime}(t) \leq \Theta$ and $0 \leq x(t) \leq \Theta t$ for $t \in I$. Moreover, by $\left(H_{2}\right),\left(H_{4}\right)$ and $\left(H_{5}\right)$, for every $t \in I$ we have

$$
\begin{aligned}
(A x)^{\prime}(t)= & \gamma \int_{0}^{1} \int_{s}^{1} f\left(\theta, x(\theta), x^{\prime}(\theta)\right) d \theta d g(s)+\int_{t}^{1} f\left(\theta, x(\theta), x^{\prime}(\theta)\right) d \theta \\
\geq & \gamma \int_{0}^{1} \int_{s}^{1} f\left(\theta, x(\theta), x^{\prime}(\theta)\right) d \theta d g(s)-\int_{t}^{1}\left|f\left(\theta, x(\theta), x^{\prime}(\theta)\right)\right| d \theta \\
\geq & \gamma \int_{0}^{1} \int_{s}^{1} f(\theta, \Theta \theta, \Theta) d \theta d g(s) \\
& \quad-\int_{0}^{1}\left(p(\theta)+q(\theta) \Phi(|x(\theta)|)+r(\theta) \Psi\left(\left|x^{\prime}(\theta)\right|\right)\right) d \theta \\
\geq & F(\Theta)-\int_{0}^{1}(p(\theta)+q(\theta) \Phi(\theta \Theta)+r(\theta) \Psi(\Theta)) d \theta \\
\geq & F(\Theta)-P(0)-Q(0)-\Psi(\Theta) R(0) \geq 0 .
\end{aligned}
$$

Now we define the open set

$$
B_{T}=\left\{x \in C_{0}^{1}(I):\|x\|_{0}^{1}<T\right\},
$$

where $T$ is the positive constant ensured by assumption $\left(H_{3}\right)$. Since, by $\left(H_{5}\right), T<\Theta$, we have $\mathbb{K} \cap B_{T} \subset \mathbb{K} \cap B_{\Theta}$ and thus

$$
A\left(\mathbb{K} \cap B_{T}\right) \subset \mathbb{K} .
$$

Furthermore, we will show that $\lambda x \neq A x$ for every $\lambda \geq 1$ and $x \in \mathbb{K}$ with $\|x\|_{0}^{1}=T$. Suppose that, on the contrary, $x \in \mathbb{K},\|x\|_{0}^{1}=T$ and $\lambda x=A x$ for 
some $\lambda \geq 1$. Then $0 \leq x^{\prime}(t) \leq T$ and $0 \leq x(t) \leq T t$ for $t \in I$. Thus, taking into account assumption $\left(H_{2}\right)$, for every $t \in I$ we have

$$
\begin{aligned}
x^{\prime}(t) \leq & \lambda x^{\prime}(t)=(A x)^{\prime}(t) \\
\leq & |\gamma| \int_{0}^{1} \int_{s}^{1}\left|f\left(\theta, x(\theta), x^{\prime}(\theta)\right)\right| d \theta d g(s)+\int_{t}^{1}\left|f\left(\theta, x(\theta), x^{\prime}(\theta)\right)\right| d \theta \\
\leq & |\gamma| \int_{0}^{1} \int_{s}^{1}\left(p(\theta)+q(\theta) \Phi(x(\theta))+r(\theta) \Psi\left(x^{\prime}(\theta)\right)\right) d \theta d g(s) \\
& \quad+\int_{0}^{1}\left(p(\theta)+q(\theta) \Phi(x(\theta))+r(\theta) \Psi\left(x^{\prime}(\theta)\right)\right) d \theta \\
\leq & |\gamma| P_{g}+|\gamma| \int_{0}^{1} \int_{s}^{1} q(\theta) \Phi(\theta T) d \theta d g(s)+|\gamma| \iint_{0}^{1} r(\theta) \Psi(T) d \theta d g(s) \\
& \quad+P(0)+\int_{0}^{1} q(\theta) \Phi(\theta T) d \theta+\int_{0}^{1} r(\theta) \Psi(T) d \theta \\
= & |\gamma|\left(P_{g}+\left(Q_{T}\right)_{g}+\Psi(T) R_{g}\right)+P(0)+Q_{T}(0)+R(0) \Psi(T) .
\end{aligned}
$$

Therefore we have

$$
T=\|x\|_{0}^{1}=\sup _{t \in I}\left|x^{\prime}(t)\right| \leq|\gamma|\left(P_{g}+\left(Q_{T}\right)_{g}+\Psi(T) R_{g}\right)+P(0)+Q_{T}(0)+R(0) \Psi(T),
$$

which contradicts $\left(H_{3}\right)$.

The above statements ensure that Theorem 1.1 is applicable and the assertion of our theorem follows.

In order to prove our second main result we need the following lemma.

Lemma 2.2. Let assumptions $\left(H_{1}\right)-\left(H_{3}\right)$ be satisfied, and suppose that $\left(H_{6}\right)$

$$
F(0)-P(0)-Q(0) \Phi(0)-R(0) \Psi(0)>0 .
$$

Then there exists $M \in(0, T)$ such that

$$
F(M)=P(0)+Q_{M}(0)+R(0) \Psi(M)+M,
$$

where $T$ is defined by $\left(H_{3}\right)$.

Proof. Define the continuous real-valued function $L$ by

$$
L(m):=F(m)-P(0)-Q_{m}(0)-R(0) \Psi(m)-m, \quad m \geq 0 .
$$

By assumption $\left(H_{6}\right)$ we have $L(0)>0$. Therefore, since $F$ and $L$ are con- 
tinuous, it is enough to show that $L(T)<0$. To this end observe that

$$
\begin{aligned}
F(T) & =\gamma \iint_{0}^{1} f(\theta, \theta T, T) d \theta d g(s) \leq|\gamma| \int_{0}^{1} \int_{s}^{1}|f(\theta, \theta T, T)| d \theta d g(s) \\
& \leq|\gamma| \int_{0}^{1} \int_{s}^{1}(p(\theta)+q(\theta) \Phi(\theta T)+r(\theta) \Psi(T)) d \theta d g(s) \\
& \leq|\gamma| P_{g}+|\gamma| \iint_{0 s}^{1} q(\theta) \Phi(\theta T) d \theta d g(s)+|\gamma| \iint_{0}^{1} r(\theta) \Psi(T) d \theta d g(s) \\
& =|\gamma|\left(P_{g}+\left(Q_{T}\right)_{g}+R_{g} \Psi(T)\right) .
\end{aligned}
$$

Hence it suffices to prove that

$$
|\gamma|\left(P_{g}+\left(Q_{T}\right)_{g}+R_{g} \Psi(T)\right)-P(0)-Q_{T}(0)-R(0) \Psi(T)-T<0,
$$

which, in view of $\left(H_{3}\right)$, is obvious.

TheOREM 2.3. Let assumptions $\left(H_{1}\right)-\left(H_{6}\right)$ be satisfied. Then there exists a solution $x \in C_{0}^{1}(I)$ of the boundary value problem (1.1)-(1.3) such that $M \leq\|x\|_{1}^{0} \leq T$, where $M$ and $T$ are defined by $(2.5)$ and $\left(H_{3}\right)$, respectively.

Proof. Our purpose is to apply Theorem 1.2. Indeed, consider the cone $\mathbb{K}$ and the set $B_{\Theta}$ as in the proof of Theorem 2.1 and define the open sets

$$
\begin{aligned}
& \Omega_{1}:=B_{M}=\left\{x \in C_{0}^{1}(I):\|x\|_{0}^{1}<M\right\} \\
& \Omega_{2}:=B_{T}=\left\{x \in C_{0}^{1}(I):\|x\|_{0}^{1}<T\right\} .
\end{aligned}
$$

Let also $A$ be the operator defined by $(2.2)$ on the cone $\mathbb{K}$. As in the proof of Theorem 2.1, we can show that

$$
A\left(\mathbb{K} \cap \bar{B}_{\Theta}\right) \subset \mathbb{K} .
$$

Since $M<T<\Theta$, we have $\mathbb{K} \cap\left(\bar{\Omega}_{2} \backslash \Omega_{1}\right) \subset \mathbb{K} \cap \bar{B}_{\Theta}$ and thus

$$
A\left(\mathbb{K} \cap\left(\bar{\Omega}_{2} \backslash \Omega_{1}\right)\right) \subset \mathbb{K} .
$$

Now consider an $x \in \mathbb{K} \cap \partial \Omega_{1}$. Then $\|x\|_{0}^{1}=M$, and following the same argument as in (2.3), we derive

$$
(A x)^{\prime}(t) \geq F(M)-P(0)-Q_{M}(0)-\Psi(M) R(0) .
$$

Therefore, taking into account (2.5) we have

$$
(A x)^{\prime}(t) \geq M=\|x\|_{0}^{1}, \quad t \in I,
$$

which means that

$$
\|A x\|_{0}^{1} \geq\|x\|_{0}^{1}, \quad x \in \mathbb{K} \cap \partial \Omega_{1} .
$$


Now consider a point $x \in \mathbb{K} \cap \partial \Omega_{2}$. Then $\|x\|_{0}^{1}=T$, and following the same argument as in (2.4) and taking into account $\left(H_{3}\right)$, we obtain

$$
\begin{aligned}
\|A x\|_{0}^{1} & =\sup _{t \in I}\left|(A x(t))^{\prime}\right| \\
& \leq|\gamma| P_{g}+P(0)|\gamma| Q_{g}(T)+Q_{T}(0)+\Psi(T)\left(|\gamma| R_{g}+R(0)\right) \leq T,
\end{aligned}
$$

i.e.

$$
\|A x\|_{0}^{1} \leq\|x\|_{0}^{1}, \quad x \in \mathbb{K} \cap \partial \Omega_{2} .
$$

The above statements ensure that Theorem 1.2 is applicable and the assertion of our theorem follows.

\section{Applications}

The sublinear case. Here we suppose that $f$ satisfies the following condition:

$\left(\widehat{H}_{2}\right) \quad$ There exist nonnegative real-valued functions $p, q, r$ in $L^{1}(I)$, not equal to zero almost everywhere and such that

$$
|f(t, u, v)| \leq p(t)+q(t)|u|+r(t)|v|
$$

for all $(u, v) \in \mathbb{R} \times \mathbb{R}$ and all $t \in I$.

This is, obviously, the case of assumption $\left(H_{2}\right)$ when $\Phi$ and $\Psi$ are both the identity functions. Then we have

$$
Q_{m}(t)=m \int_{t}^{1} \theta q(\theta) d \theta, \quad\left(Q_{m}\right)_{g}=m \int_{0}^{1} \int_{s}^{1} \theta q(\theta) d \theta d g(s),
$$

and thus assumption $\left(H_{3}\right)$ takes the form:

$\left(\widehat{H}_{3}\right) \quad$ There exists $T>0$ such that

$$
|\gamma|\left(P_{g}+T \int_{0}^{1} \int_{s}^{1} \theta q(\theta) d \theta d g(s)+T R_{g}\right)+P(0)+T \int_{0}^{1} \theta q(\theta) d \theta+T R(0)<T
$$

Now we set

$$
\widehat{q}(t)=t q(t), \quad \widehat{Q}(t)=\int_{t}^{1} \widehat{q}(s) d s
$$

and by (3.1) we obtain

$$
T\left(1-|\gamma| \widehat{Q}_{g}-|\gamma| R_{g}-\widehat{Q}(0)-R(0)\right)>|\gamma| P_{g}+P(0) .
$$

Then, if

$$
|\gamma|\left(\widehat{Q}_{g}+R_{g}\right)+\widehat{Q}(0)+R(0)<1
$$


we can take

$$
T>\frac{|\gamma| P_{g}+P(0)}{1-|\gamma|\left(\widehat{Q}_{g}+R_{g}\right)-\widehat{Q}(0)-R(0)}=: K
$$

Moreover assumptions $\left(H_{5}\right)$ and $\left(H_{6}\right)$ become:

$\left(\widehat{H}_{5}\right) \quad$ there exists $\Theta>K$ such that $F(\Theta)-P(0)-Q_{\Theta}(0)-\Theta R(0) \geq 0$, and

$$
F(0)>P(0)
$$

respectively.

Therefore we have the following corollaries of Theorems 2.1 and 2.3 respectively.

Corollary 3.1. Let assumptions $\left(H_{1}\right),\left(\widehat{H}_{2}\right),\left(H_{4}\right),\left(\widehat{H}_{5}\right)$ be satisfied, and suppose that (3.2) holds. Then there exists a solution $x \in C_{0}^{1}(I)$ of the boundary value problem (1.1)-(1.3) such that $0 \leq x(t) \leq T t$ for $t \in I$, where $T>K$ and $K$ is defined by $(3.3)$.

Corollary 3.2. Let assumptions $\left(H_{1}\right),\left(\widehat{H}_{2}\right),\left(H_{4}\right),\left(\widehat{H}_{5}\right),\left(\widehat{H}_{6}\right)$ be satisfied, and suppose that (3.2) holds. Then there exists a solution $x \in C_{0}^{1}(I)$ of the boundary value problem (1.1)-(1.3) such that $M \leq\|x\|_{1}^{0} \leq T$, where $T>K$ and $K, M$ are defined by (3.3), (2.5), respectively.

An example. Consider the following nonlocal boundary value problem:

$$
\begin{aligned}
& x^{\prime \prime}(t)+b x^{\prime}(t)+b t x(t)-1=0, \quad t \in I, \\
& x(0)=0, \quad x^{\prime}(1)=\int_{0}^{1} x^{\prime}(s) d g(s),
\end{aligned}
$$

where $b$ is a positive constant and $g(s)=\frac{5}{4} s^{2}$.

We wish to check the applicability of Corollary 3.2 to this boundary value problem. In the present case we have $f(t, u, v)=-1+b t u+b v$ and thus $|f(t, u, v)| \leq 1+b|u|+b|v|$. So it is clear that assumptions $\left(H_{1}\right),\left(H_{2}\right)$ are satisfied, and moreover, $p(t)=1$ and $q(t)=r(t)=b$. Furthermore we have

$$
\gamma=-4, \quad F(0)=-4 \int_{0}^{1} \int_{s}^{1}(-1) d \theta d g(s)=\frac{5}{3}, \quad P(0)=\int_{0}^{1} p(s) d s=1 .
$$

Observe that $F(0)=5 / 3>1=P(0)$, so assumption $\left(\widehat{H}_{6}\right)$ is satisfied. Also, since $\gamma f(t, u, v)=4-4 b t u-4 b v, \gamma f$ is nonincreasing with respect to the variables $u, v$, i.e. assumption $\left(H_{4}\right)$ is satisfied. Moreover, we have

$$
\widehat{Q}(t)=\int_{t}^{1} b s d s=\frac{1-t^{2}}{2} b, \quad \widehat{Q}_{g}=\int_{0}^{1} \widehat{Q}(s) d g(s)=\frac{5 b}{16},
$$




$$
R(t)=(1-t) b, \quad R(0)=b, \quad R_{g}=\frac{5 b}{12} .
$$

Thus, inequality (3.2) takes the form $b<12 / 53$. Moreover we have

$$
P(t)=1-t, \quad P_{g}=\frac{5}{12}
$$

and thus, since $b<12 / 53$, by $(3.3)$ we obtain

$$
T>\frac{32}{12-53 b} \text {. }
$$

It remains to prove that assumptions $\left(\widehat{H}_{3}\right),\left(\widehat{H}_{5}\right)$ hold. To do this we observe that

$$
\begin{gathered}
F(\Theta)=\gamma \int_{0}^{1} \int_{s}^{1}\left(-1+b \theta^{2} \Theta+b \Theta\right) d \theta d g(s)=\frac{5-8 b \Theta}{3}, \\
P(0)=1, \quad Q_{\Theta}(0)=\frac{b \Theta}{2}, \quad \Psi(\Theta)=\Theta
\end{gathered}
$$

Hence $\left(\widehat{H}_{5}\right)$ becomes:

$$
\text { there exists } \Theta>K=\frac{32}{12-53 b} \text { such that } \Theta \leq \frac{4}{25 b} \text {. }
$$

Finally, using (2.5), we deduce that $M=4 /(25 b+6)$.

Since we must have $M<T<\Theta$, we must also have

$$
\frac{4}{25 b+6}=M \leq \frac{32}{12-53 b}<T<\Theta \leq \frac{4}{25 b} .
$$

It is clear that this inequality can hold if $0<b<48 / 1022$. In conclusion, if $0<b<48 / 1022$, then all assumptions of Corollary 3.2 are satisfied, and thus we have proved the following result:

If $0<b<48 / 1022$, then there exists a solution $x \in C_{0}^{1}(I)$ of the boundary value problem (3.4), (3.5) such that

$$
\frac{4}{25 b+6} \leq\|x\|_{1}^{0}<\frac{4}{25 b} \text {. }
$$

\section{References}

[1] R. P. Agarwal and D. O'Regan, Singular differential, integral and discrete equations: The semipositone case, Moscow Math. J. 2 (2002), 1-15.

[2] R. P. Agarwal, D. O'Regan and P. J. Y. Wong, Positive Solutions of Differential, Difference and Integral Equations, Kluwer, Dordrecht, 1999.

[3] H. Amann, Fixed point equations and nonlinear eigenvalue problems in ordered Banach spaces, SIAM Rev. 18 (1976), 620-709. 
[4] V. Anuradha, D. D. Hai and R. Shivaji, Existence results for superlinear semipositone BVP's, Proc. Amer. Math. Soc. 124 (1996), 757-763.

[5] D. Cao and R. Ma, Positive solutions to a second order multi-point boundary-value problem, Electron. J. Differential Equations 2000, no. 65, 7-8.

[6] A. Castro and R. Shivaji, Non-negative solutions for a class of non-positone problems, Proc. Roy. Soc. Edinburgh Sect. A 118 (1998), 291-302.

[7] D. R. Dunninger and H. Wang, Multiplicity of positive solutions for a nonlinear differential equation with nonlinear boundary conditions, Ann. Polon. Math. 69 (1998), 155-165.

[8] P. W. Eloe, J. Henderson and P. Wong, Positive solutions for two-point boundary value problems, in: Proc. Dynamic Systems and Applications, Vol. 2 (Atlanta, GA, 1995), Dynamic, Atlanta, GA, 1998, 135-144.

[9] L. H. Erbe, S. Hu and H. Wang, Multiple positive solutions of some boundary value problems, J. Math. Anal. Appl. 184 (1994), 640-648.

[10] L. H. Erbe and H. Wang, On the existence of positive solutions of ordinary differential equations, Proc. Amer. Math. Soc. 120 (1994), 743-748.

[11] D. J. Guo and V. Lakshmikantham, Nonlinear Problems in Abstract Cones, Academic Press, San Diego, 1988.

[12] Y. Guo, W. Ge and Y. Gao, Twin positive solutions for higher order m-point boundary value problems with sign changing nonlinearities, Appl. Math. Comput. 146 (2003), 299-311.

[13] C. Gupta, Solvability of a generalized multi-point boundary value problem of mixed type for second order ordinary differential equations, in: Proc. Dynamic Systems and Applications, Vol. 2 (Atlanta, GA, 1995), Dynamic, Atlanta, GA, 1998, 215-222.

[14] C. Gupta, S. K. Ntouyas and P. Ch. Tsamatos, On an m-point boundary value problem for second order ordinary differential equations, Nonlinear Anal. 23 (1994), $1427-1436$.

[15] J. Henderson and H. Wang, Positive solutions for nonlinear eigenvalue problems, J. Math. Anal. Appl. 208 (1997), 252-259.

[16] V. Il'in and E. Moiseev, Nonlocal boundary value problems of the second kind for a Sturm-Liouville operator, Differ. Equ. 23 (1987), 979-987.

[17] G. L. Karakostas and P. Ch. Tsamatos, Positive solutions for a nonlocal boundaryvalue problem with increasing response, Electron. J. Differential Equations 2000, no. $73,1-8$.

[18] - - - Multiple positive solutions for a nonlocal boundary value problem with response function quiet at zero, ibid. 2001, no. 13, 1-10.

[19] - - - Sufficient conditions for the existence of nonnegative solutions of a nonlocal boundary value problem, Appl. Math. Lett. 15 (2002), 401-407.

$[20]-,-$, Functions uniformly quiet at zero and existence results for one-parameter boundary value problems, Ann. Polon. Math. 88 (2002), 267-276.

[21] M. A. Krasnosel'skiı̌, Positive Solutions of Operator Equations, Noordhoff, Groningen, 1964.

[22] Y. Li, Positive solutions for second order boundary value problems with sign changing nonlinear terms, J. Math. Anal. Appl. 282 (2003), 232-240.

[23] P. L. Lions, On the existence of positive solutions of semilinear elliptic equations, SIAM Rev. 24 (1982), 441-467.

[24] B. Liu, Positive solutions of a nonlinear three-point boundary value problem, Comput. Math. Appl. 44 (2002), 201-211.

[25] R. Ma, Positive solutions for a nonlinear three-point boundary-value problem, Electron. J. Differential Equations 1998, no. 34, 1-8. 
[26] R. Ma and H. Wang, Positive solutions of nonlinear three-point boundary-value problems, J. Math. Anal. Appl. 279 (2003), 216-227.

[27] H. Wang, On the existence of positive solutions for semilinear elliptic equations in the annulus, J. Differential Equations 109 (1994), 1-4.

Department of Mathematics

University of Ioannina

45110 Ioannina, Greece

E-mail: ptsamato@cc.uoi.gr

Reçu par la Rédaction le 20.10.2003

Révisé le 22.1.2004 